$\S=-1$

\title{
Optical Sensor for Analysis of Amonia, Iron and Manganese Nutrients Pranav B Lapsiwala ${ }^{1}$ *
}

\author{
${ }^{1}$ Sarvajanik College of Engineering \& Technology \\ *Corresponding author E-mail:pranav.lapsiwala@gmail.com
}

\begin{abstract}
The soil nutrients sensor is developed with the help of Light Emitting diodes (LEDs), Photodiode, microcontroller or microprocessor circuit and data logger system. The sensor is working on photometric principle and detects the soil nutrients. The wavelength of LED used as chemical agent and that is reacts with soil sample. The wavelength of LED is useful to detect nutrients like ammonia nitrogen (NHf4-N), iron (Fe), manganese (Mn) by colour changes with the help of different chemical reagent. The resolution of 1.0-20 mg/100 g has been performed for solution of soil nutrients. The samples are taken from different land and results are compared with database created for analysis.
\end{abstract}

Keywords: soil nutrient, optical sensor, light emitting diode, photometric.

\section{Introduction}

To check the soil fertility nutrient of the soil must be known and that help farmer for quality and quantity used of fertilizer. The known soil condition is very important not only for effective production but also avoid underwater pollution by different fertilizers. The laboratory testing and analysis method is costly and time consuming. Thus, simple and inexpensive methods of measurement of soil nutrients are required. A simple method of photometer reflection has been used to detect nitrate, iron and manganese in soil. This system becomes a useful tool for a quantitative analysis of soil nutrients. However, detection of more nutrients is highly desirable. The colour developing chemical method for testing and analysis of soil is generally used by farmers by giving the sample to laboratory and get the results. Solutions of nutrients extracted from a soil, whose colour is developed by chemical reagent, are estimated by a subjective judgment with the colour chart. But due to variation of soil content gives fluctuating analysis. For precise measurement spectrophotometer can be applied to investigate the colour developed in solutions. However, spectrophotometer is expensive and complex.

To overcome the problems, LEDs based optical sensor is proposed to analyze colour based soil nutrient analyser. LEDs are low cost, smaller in size and above all having wide range of operation from ultraviolet to infrared. Therefore, it is easy to get LED for specific band of absorption.

In this article, experiment work of Optical Sensor consists of LEDs source, microcontroller and photodiode used. The quantitative analysis of soil nutrient is performed using soil analyser.

\section{Absorption Spectra of Colour Development Reagent}

To decide the wavelength of LED for the sensor, absorption spectra of colour developed standard solution studied and given in Table 1.
Table 1: Standard database for soil nutrients

\begin{tabular}{|c|c|c|}
\hline & Content $(\mathbf{m g} / \mathbf{1 0 0} \mathbf{g})$ & Colour char $(\mathbf{m g} / \mathbf{1 0 0} \mathbf{g})$ \\
\hline $\mathrm{NH}_{4}-\mathrm{N}$ & $5,10,15,20,25$ & $1,5,10,15,20,25$ \\
\hline $\mathrm{Fe}\left(\mathrm{mg} \mathrm{Kg}^{-1}\right)$ & $5,10,25,50,75,100$ & $5,10,25,50,75,100$ \\
\hline $\mathrm{Mn}\left(\mathrm{mg} \mathrm{Kg}^{-1}\right)$ & $5,10,25,50,75,100$ & $5,10,25,50,75,100$ \\
\hline
\end{tabular}

An absorption spectrum for ammonia nitrogen (NH4-N) with different content is shown in figure 1(a). For the ammonia nitrogen determination, the Indophenol method is applied and the sample solution become green with an absorption peak achieve at $650 \mathrm{~nm}$. The iron $(\mathrm{Fe})$ and manganese $(\mathrm{Mn})$ are analysed by 2,4,6 - tripyridlyl - 1,3,5 - triazine (TPTZ) method. The colour developed sample of iron $(\mathrm{Fe})$ becomes purple and having absorption peak at 600 $\mathrm{nm}$ in figure $1(\mathrm{~b})$ while manganese solution become pink and having absorption peak to $530 \mathrm{~nm}$ show in figure 1(c).

\section{Sensor Configuration}

All title and The absorption spectra gives detail about the wavelength and based on that Red and Green LED are selected. The emission spectra of LED are measured on Optical Spectrum Analyser and the measured center wavelength of Red LED is $632 \mathrm{~nm}$ and Green LED is $524 \mathrm{~nm}$.

The developed sensor having two light source of green $(\mathrm{G})$ and red (R) package is polished and glued to one end of a $40 \mathrm{~mm}$ long plastic optical fiber (POF) with a diameter of $1.0 \mathrm{~mm}$. The light coupled into one of the end of glued couple POF and transmitted into a transparent plastic cell and then detected by silicon photodiode having detecting area $10 \times 10 \mathrm{~mm} 2$. The output of the photodiode fed to the ADC pin of microcontroller circuit. The microcontroller circuit interfaces with PC via RS-232 and provide a signal and data processing capabilities.

The LEDs are sequentially operated for $0.3 \mathrm{~s}$ and output of the photodiode is averaged over each LED operation period in the microcontroller circuit and send to the PC via RS 232 interface. Therefore, the transmitted light intensities from the LEDs are obtained in one measurement. 


\section{Experiment Results and Discussion}

\subsection{Experiment of Soil Sample}

The sensor was tested with colour developed standard solution given in table 1 . The transmitted intensity of different content was measured at room temperature. Ten, successive measurement of the same sample were performed, and averaged value used for the data. Figure 2(a) - (c) shows the result with the LED giving the largest sensitivity and the colour of the LED is shown in figure. The normalized sensor output plotted by a solid circle together with its least-squares fitting curves.

\subsection{Theoretical Consideration}

The transmitted intensity over individual sample for an individual spectral component of LED with sensor output $I_{o}$ is given by

$I_{0}=\sum_{k=1}^{n} \mathrm{I}_{\mathrm{L}}\left(A_{\mathrm{k}}\right) \exp \left\{-\alpha\left(A_{\mathrm{k}}\right) \mathrm{L}\right\} \mathrm{n}\left(A_{\mathrm{k}}\right)$

Where $I_{L}\left(\kappa_{k}\right)$ is the normalized intensity of LED, $\alpha\left(K_{k}\right)$ is the absorption coefficient of the colour developed standard database, $\eta\left(\kappa_{k}\right)$ is the normalized spectral sensitivity of the photodiode at a wavelength $\left(\kappa_{k}\right)$. The absorption coefficient at given wavelength $\left(\kappa_{k}\right)$ is calculated from figure 1 . To solve the equation 1 , the interval of wavelength is $2.0 \mathrm{~nm}$ for both colour LED is taken. The wavelength range of green LED is $500-550 \mathrm{~nm}$ and $610-660 \mathrm{~nm}$ is applied. The value of $\eta\left(\kappa_{k}\right)$ is taken from the photodiode specification for each wavelength range. The calculated value of sensor output $I_{o}$ is normalized and shown in figure 2 with an open circle. From the figure 2 it is depict that there is good agreement between theory and practical results. In addition, calculations are closely predicted and can be applied to other wavelength range also. To validate the sensor output on the variation of LED wavelength, the wavelength of $\mathrm{LED}_{\mathrm{s}}$ changes $\pm 10 \mathrm{~nm}$ from its original value without changing the spectral profile. The procedure is same as above for theoretical calculation of sensor output is applied and that plotted in figure 3 with original value of $I_{o}$. It is observed that there is relatively large deviation found in $\mathrm{F}_{\mathrm{e}}$, and due to the emission band of green LED does not match with absorption peak of $F_{e}$ solution.

Table 2: Normalized intensity (x) with content of (y) in figure2

\begin{tabular}{|l|l|l|}
\hline \multicolumn{3}{|c|}{$\mathrm{y}=\mathrm{a}(\boldsymbol{x})+b$} \\
\hline & $\mathrm{a}$ & $\mathrm{b}$ \\
\hline $\mathrm{NH} 4-\mathrm{N}$ & -8.50 & -0.15 \\
\hline $\mathrm{Fe}$ & -44.85 & -2.10 \\
\hline $\mathrm{Mn}$ & -345.5 & -0.50 \\
\hline
\end{tabular}

For above experiment ten soil sample were taken from a farmland (sample 1,2), green field (sample 3-8) and rice field (9,10). The content of soil nutrient is evaluated using the fitting curve for standard database and parameter listed in table 2. In addition, for the more colour developed solutions, the results are strongly development on lighting method and therefore sensor output gives misjudgment is obtained results. However, more samples can give accuracy in sensor results.

\section{Conclusion}

In this section you The article is represents the LED, photodiode and microcontroller based optical sensor for soil nutrient analysis is discussed. The sensing characteristic of LED sources is investigated on ten different soil samples and their theory and experiment analysis shown for validation of prediction. The chemical reagent used for generating colour sample is sensitively detects by proposed sensor for measurement of soil nutrients. The achieved result is as small as $1.0-2.0 \mathrm{mg} / 100 \mathrm{~g}$ for ammonia nitrogen $(\mathrm{NH} 4-\mathrm{N})$,
2.0 - $2.5 \mathrm{mg} \mathrm{Kg}-1$ for iron $(\mathrm{Fe})$ and Manganese $(\mathrm{Mn})$ for the standard solution of soil nutrients database. There are good result agreement found between theory and experiment result of absorption spectra of LEDs and colour developed standard database. The ten sample of different field is taken and their result is compared with standard colour chart and found that sensor having slight difference of colour and misjudgment happening in the colour chart decision.

\section{References}

[1] M. Toole, D. Diamond "Absorbance Based Light Emitting Diode Optical Sensors and Sensing Devices", Soil Science Society of America Journal, 2008, pp. 2453-2479.

[2] E. Ben-Dor, A. Banin "Near-Infrared Analysis as a Rapid Method to Simultaneously Evaluate Several Soil Properties", Soil Science Society of America Journal, 1985, pp.364-37.

[3] R. Dalal, R. Henry "Simultaneous determination of moisture, organic carbon and total nitrogen by near infrared reflectance spectrophotometry", Science Society of America Journal, 1986, pp.120-123.

[4] S.J. BIRRELL "Multi-sensor ISFET system for soil analysis", Science Society of America Journal, 1993, pp.1-9.

[5] S. Birrell, J. Hummel "Membrane selection and ISFET configuration evaluation for soil nitrate sensing", American Society of Agricultural Engineers, 2000, pp. 197-206.

[6] C. Cheng, D. Laird, "Near-infrared reflectance spectroscopy- principal components regression analyses of soil properties", Science Society of America Journal, 2001, pp.480-490.

[7] B. Stenberg, A. Jonsson "Near infrared technology for soil analysis with implications for precision agriculture", Swedish University of Agricultural Sciences, 2002, pp.279-284.

[8] B. Jahn, S. Upadhyay "Wavelet-based spectral analysis for soil nitrate content measurement", Science Society of America Journal, 2005, pp.2065-2071.

[9] S. Stamatiadis, V. Samaras et al "Ground-sensor soil reflectance as related to soil properties and crop response in a cotton field", Precision Agriculture, 2005, pp.399-411.

[10] M. Nanni , M. Dematte "Spectral reflectance methodology in comparison to traditional soil analysis", Soil Science Society of America Journal, 2006, pp.393-404.

[11] S. Lemos, J. Alonso et al "Soil calcium and $\mathrm{pH}$ monitoring sensor system", Journal of agricultural and food chemistry, 2007, pp.4658-4663.

[12] R. Rinnan, A. Rinnan "Application of near infrared reflectance (NIR) and fluorescence spectroscopy to analysis of microbiological and chemical properties of arctic soil", Soil Biology and Biochemistry, 2007, pp.1 664-1673.

[13] E. Dor, D. Heller et al "A novel method of classifying soil profiles in the field using optical means", Soil Science Society of America Journal , 2008,pp. 1113-1123.

[14] H. Kim, J. hummel, "Soil macronutrient sensing for precision agriculture", Journal of Environmental Monitoring, 2009, pp.3047-3310.

[15] C. Due, J. Zhou "Application of Infrared Photoacoustic Spectroscopy in Soil Analysis", Applied Spectroscopy Reviews, 2011, pp.405422

[16] H. MAhmood, J. Henten et al "Sensor data fusion to predict multiple soil properties", Precision Agriculture, 2012, pp.628-645.

[17] X.An, H. Sun "A portable soil nitrogen detector based on NIRS", Precision Agriculture, 2014, pp.3-16.

[18] P. Han, D.Dong et al "A smartphone-based soil color sensor: For soil type classification", Computers and Electronics in Agriculture , 2016, pp.232-241. 


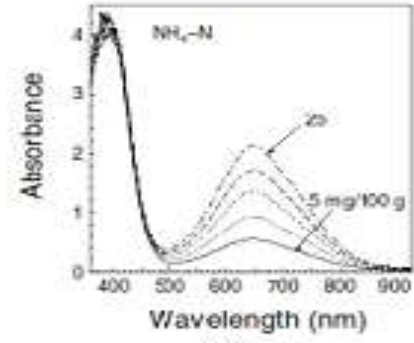

(a)

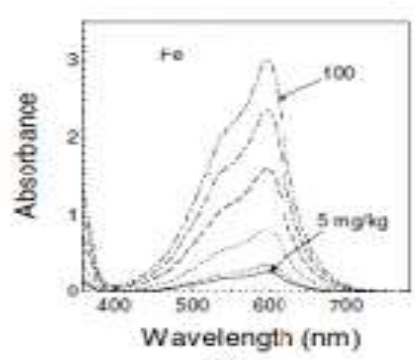

(b)

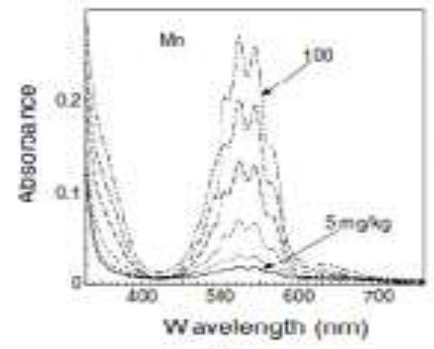

(c)

Fig 1: Absorption Spectra Colour develop solution with various content of soil nutrients: (a) $\mathrm{NH}_{4}-\mathrm{N}$, (b) $\mathrm{F}_{\mathrm{e}}$, (c) $\mathrm{M}_{\mathrm{n}}$

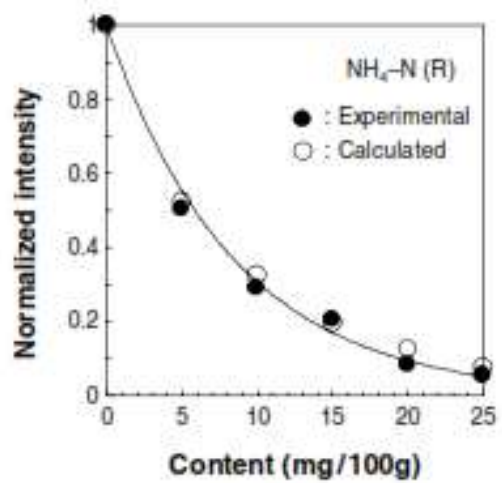

(a)

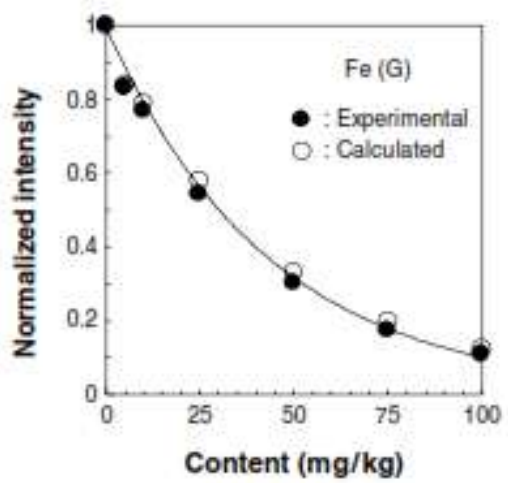

(b)

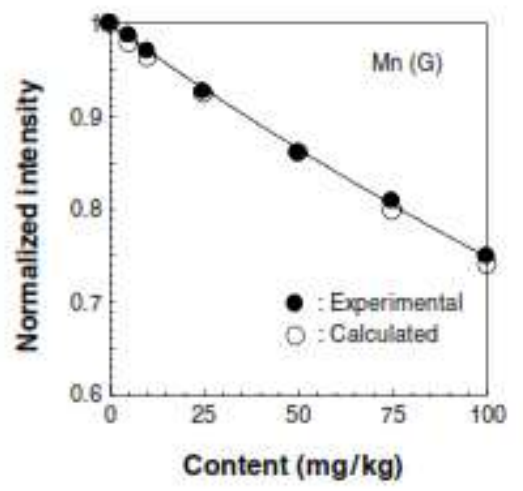

(c)

Fig 2 : The transmitted intensity of LED light on the content of soil nutrients: (a) $\mathrm{NH}_{4}-\mathrm{N}$, (b) $\mathrm{F}_{\mathrm{e}}$, (c) $\mathrm{M}_{\mathrm{n}}$ 


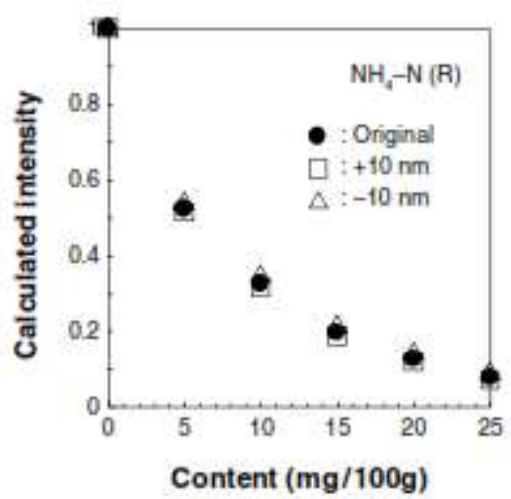

(a)

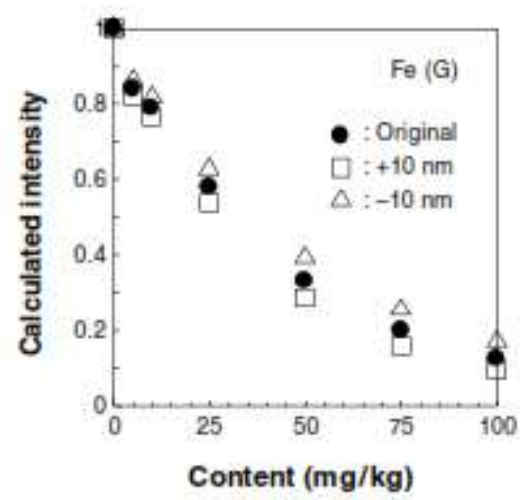

(b)

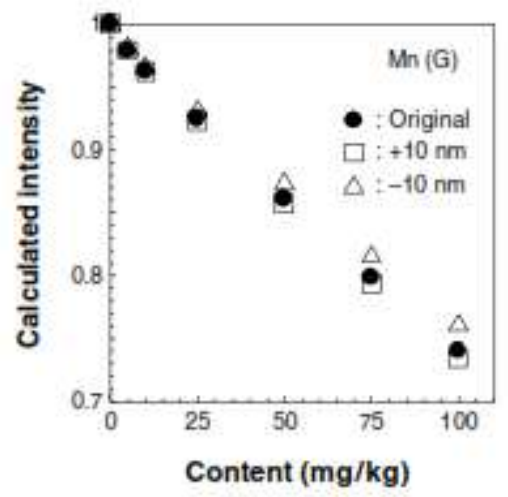

(c)

Fig 3: Analysis of LED light for soil sample with and without wavelength shift of $\pm 10 \mathrm{~nm}$ 